\title{
Study of Network-Centric Maintenance Intelligent Decision Technology Based SOA
}

\author{
Hongyu $\mathrm{Ge}^{1, \text { a }}$, Quan $\mathrm{Shi}^{1}$, Wei Xia ${ }^{1}$ and Zhifeng You ${ }^{1}$ \\ ${ }^{1}$ Department of Equipment Command \& Management, Mechanical Engineering College, \\ Shijiazhuang 050003, China; \\ aghy_paper@163.com
}

Keywords: SOA, intelligent, decision making.

\begin{abstract}
For the development of modern information technology, take the advanced concepts and new technologies, new methods of foreign maintenance aspects as lessons, adopt a network-centric technology to build SOA-based network maintenance intelligent decision technology. This paper introduces network maintenance and support SOA-based architecture, proposes network maintenance intelligent decision technology and its component, it is instructive for the construction of the SOA-based network maintenance.
\end{abstract}

\section{Introduction}

SOA is short for Service Oriented Architecture, is a service-oriented architecture, one kind of business driven IT architecture, a component model, supporting the integration of various business, achieving business standardization work, service-oriented and component[1], the SOA packaging different functional units of the system as services, it can complete transformation of state information or business data, make it change from a valid or consistent state to another, the service can interact with a unified and generic way, it is suitable for reusable components in the various business process applications, Prevent data isolated island at the beginning of the service, improving the processing efficiency of information and shorten the time of repair application response, promoting the production of complete maintenance support program, Improving equipment maintenance efficiency[2].

SOA as a new method of software architecture, based on open standards and protocols, with loosely coupled, support the efficient integration of applications and business processes on-demand features, to achieve the flexibility and functionality of the system scalability, at the same time, it can integrate process-oriented business when develop the SOA architecture. Using SOA can transform from the distributed, complex, inflexible heterogeneous resources to the integrated, simplified and highly flexible resources, and adjust this kind of resource, in order to directly support the related business[3]. From various maintenance data sharing and various maintenance information integration and the needs of application system, SOA provides a way and a new concept for network system design, it is a effective method for network maintenance architecture development.

\section{Maintenance Intelligent Decision Technology}

Maintenance of network-centric information technology network platform and equipment maintenance support information systems as the basis, achieving the data integration and transmission from single to group, so as to provide information and decision support for equip security departments to grasp the time repairs, planned maintenance, condition-based maintenance, battlefield damage assessment and repair. However, according to the dynamic usage of equipment, health conditions, faults and damage information, resource consumption information service request information on how to repair the equipment for scientific decision-making, thereby generating equipment maintenance programs, resource availability and resource optimization program scheduling scheme, and implement maintenance and support activities, it is the problem of network-centric maintenance which need to be solved, in order to improve the efficiency of 
maintenance and support decision-making, science and rationality, artificial intelligence techniques can be used to solve the network-centric intelligent decision maintenance problems. The artificial intelligence is a comprehensive subject which is a mutual penetration of computer science, cybernetics, information theory, neurophysiology, psychology, linguistics and other subjects[4]. Program system which is an expert system with a lot of expertise and experience in the application of artificial intelligence and computer technology, based on the knowledge and experience of a procedural system of one or more experts in the field of reasoning and judgment, simulate human experts decision-making process, in order to solve complex problems that require human experts handle. For smart decision-making process in Figure2.

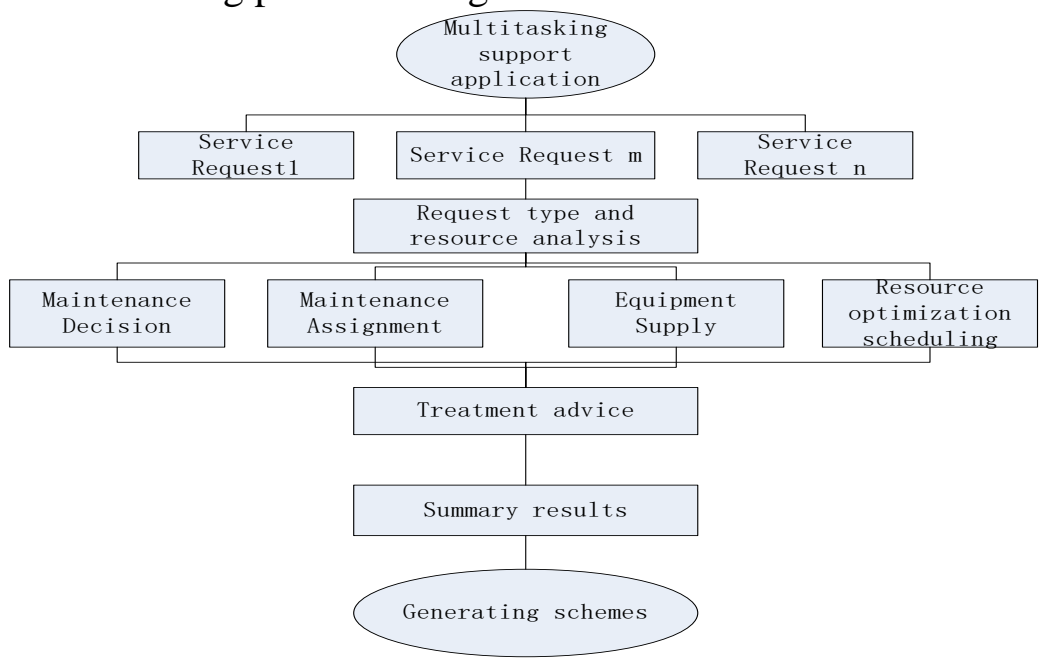

Fig. 1 Smart decision-making process

From the view of the structural point, intelligent decision-making process can be abstracted as a black-box model, damage to equipment and other data parameters entered into the intelligent decision to apply the model to generate a complete technical support service program through the decision-making, staff scheduling, resource allocation, and some other column-related programs, reducing the waiting time to repair, greatly improving the efficiency of equipment maintenance.

\subsection{The Generation Technology Based On Intelligent Maintenance Program.}

Maintenance program is to produce a maintenance program on the basis of documents, on the basis of the maintenance program, through the integration of the maintenance information, inputting to the application program for equipment maintenance intelligent generation system, through intelligent system analysis, produce specific and detailed maintenance program. Simple process is as follows, the integrated use of rule-based reasoning, reasoning based reasoning and model-based, get smart decisions maintenance program knowledge acquisition and representation, according to the equipment to make use of the situation, health status, fault and damage information, based on equipment maintenance program and outline BDAR[5], automated generation maintenance program through the establishment of an integrated reasoning mechanisms (including the timing of maintenance and repair projects, maintenance tasks, etc.), enhancing the maintenance program to generate scientific rationality.

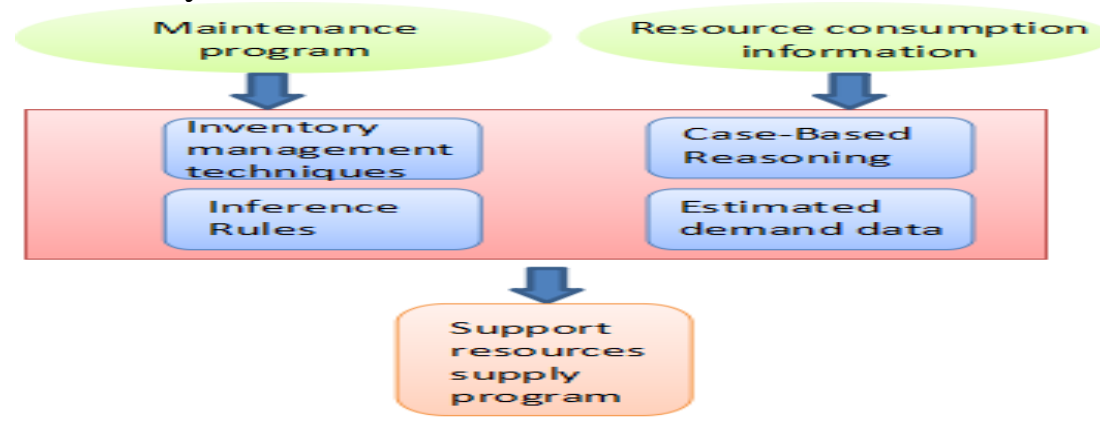

Fig. 2 Intelligent maintenance program 


\subsection{Rule-Based Generation Technology of Support Resources Supply Program}

According to the consumption information of the maintenance programs and resources, integrated using inventory management techniques, case-based reasoning technology and rule-based reasoning [6], on the base of the support resource require data, establishing integrated Reasoning mechanism, intelligent supply program to generate support resources.

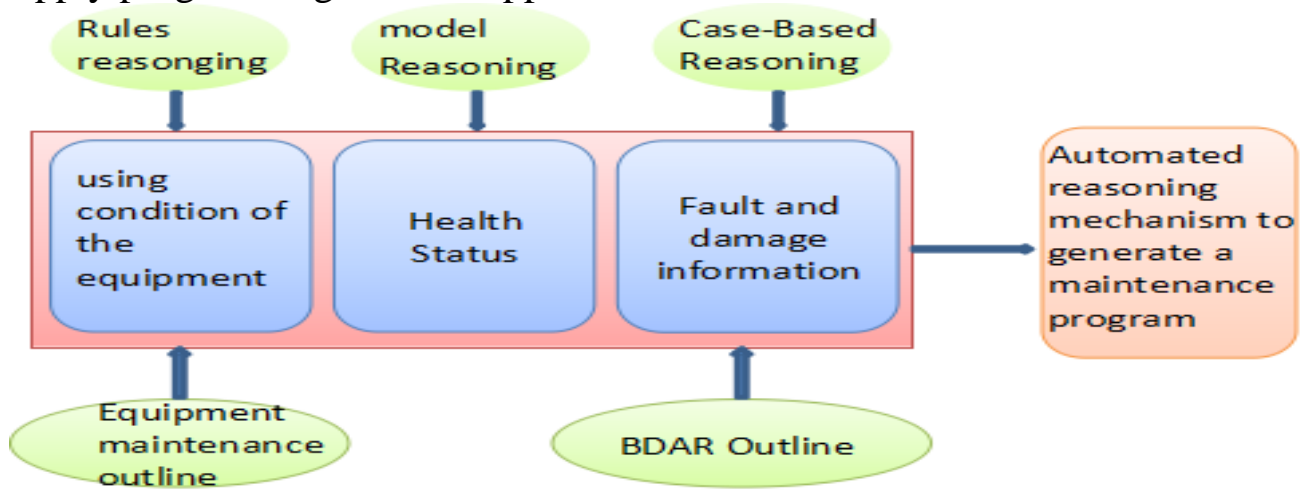

Fig. 2 Resources supply program

network-centric intelligent maintenance decisions is the basis of the getting inference rules. Whether the knowledge is complete and consistent, the expression of accurate, flexible, organizational knowledge is reasonable, for the maintenance of network-centric intelligent decision reasoning is tight, accurate will have a major impact, according to the network-centric maintenance service requests, maintenance and support decision-making rules designed to classify, Constituting a set of rules include:

RULE(MSMM): maintenance and support tasks matching rule base, analyzed by maintenance support task requests can learn what resources are necessary to perform the task, further clear division of tasks. Then according to this judgment result, can match the specific data query and service request information from the database required by the rules of inference. Such as equipment failure, issuing equipment supply request information, system analyzes this information, this task can be determined as the primary equipment supply mission, and then after the service request report extract request information to deduce equipment supply and replenishment rule base;

RULE (MDMM): maintenance decision model library. Including corrective maintenance, planned maintenance, condition-based maintenance and BDAR, and other aspects of battlefield decision model for the service request maintenance support information model solution to provide a scientific basis for decision-making for maintenance;

RULE (RRMA): maintenance and support tasks assignment rule base. there is different between the maintenance and support functions, advantages of the technical level, and power allocation, how to make the task assignment accurately and quickly according to the service request is one of the important issues of concern to equip security agencies. Through the maintenance and support task rule base will be able to automate tasks assignment maintenance and support, reducing the human aspects of the operation, the task assigned to improve science and rationality;

RULE (MMS): equipment supply and replenishment rule base. According to spare parts inventory control strategy, according to equipment supply requested information on the type and quantity of equipment supply automatically calculated to produce equipment supply security programs.

RULE (FTD): optimal scheduling rule base of support resources. For example, there is no requirement to apply for maintenance support resources in inventory, or when they were occupied by other applicants, according to support optimal scheduling rule base resource scheduling horizontal or apply to the higher security institutions.

\subsection{Maintenance Resources Based on Dynamic Programming Techniques to Optimize Scheduling}

Dynamic programming is a branch of operations research, which is to solve the problem of multi-stage decision optimization method, since the advent of dynamic programming is widely used in the allocation of resources and production planning cargo transportation and other storage areas, 
which put a complex system analysis problem into a multi-stage decision-making process, according to a certain order or sequence, beginning from the first stage, determine the optimal strategy for each successive stage[7]. In a network-centric maintenance, there is large demand for repair services and has greater randomness, need to request to achieve maintenance resource scheduling. Considering two maintenance services on the basis of demand uncertainty, giving the formal description of maintenance resource optimization scheduling problem, constructing a dynamic programming mode for the service request[8], and with the stochastic dynamic programming principle and dynamic programming algorithm based on the model predictions, establish the objective function of the decision-making process and realizing maintenance resources scheduling.

\section{Conclusion}

Intelligent Decision is a comprehensive integrated approach, there are many factors in equipment maintenance intelligent decision-making process, how to efficiently complete the development of maintenance programs and maintenance personnel assignment under these factors are the problems of maintenance decision. This paper introduces the technology of maintenance intelligent decision, tells that how to solve the three problems of maintenance program development, produce supply scheme, optimizing maintenance resources, its high technical feasibility providing a strong basis for the development of the future SOA-based network service technicians.

\section{References}

[1]. Joonseok PARK, Mikyeong MOON, Keunhyuk YEOM.. VARIABILITY MODELING TO DEVELOP FLEXIBLE SERVICE-ORIENTED APPLICATIONS. Journal of Systems Science and Systems Engineering. (2011) No. 2, p. 193-216.

[2]. Huimin Zhang, Hairong Hu, Weining Cui, et al. Study and Use of Data Synchronization in the General Equipment Support Information System Based on SOA. Journal of Sichuan Ordnance. (2013)No.7,p.63-66

[3]. Wen Ye. Application of SOA Architecture in Information System. Computer Knowledge and Technology. (2009)No.32,p.8873-8874

[4]. Guihua Nie, Xilin Tang. Research on the Application of AI and Optimization for Decision Process. journal of Wuhan University of Technology. (2000)No.5,p.135-136

[5]. Fei Lin. Preliminary Analysis of Maintenance Program and Maintenance Project. Jiangsu Aviation. (2005)No.5,p.32-33.

[6]. Bo He, Quanli Liu, Yue Wang, et al. Fault Diagnosis Expert System Based on Mixed Reasoning Mechanism. Microcomputer Information. (2006)No.25,p.192-194

[7]. Bohui Wang, Ligang Ai, Zhuo Sun. the problem of water delivering based on dynamic programming method. Water Conservancy Science and Technology and Economy. (2011)No.4,p.33-35

[8]. Jing Liu, Jifeng He, Zhiming Liu. A strategy for service realization in service-oriented design. Science in China. (2006)No.6,p.864-884 